\title{
Interpretability and accuracy issues in evolutionary multi-objective fuzzy classifiers
}

\author{
Praveen Kumar Shukla* \\ Department of Information Technology, \\ Babu Banarasi Das Northern India Institute of Technology, \\ Lucknow, India \\ Email: praveenshuklaniec@yahoo.co.in \\ *Corresponding author
}

\section{Surya Prakash Tripathi}

Department of Computer Science and Engineering, Institute of Engineering and Technology,

Lucknow, India

Email: tripathee_sp@yahoo.co.in

\begin{abstract}
Fuzzy systems have remarkable capability to deal with imprecise and uncertain information existing in the real world complex problems. Evolutionary approaches, i.e., genetic algorithms are utilised to improvise the designing of fuzzy systems. During the design of fuzzy systems, interpretability and accuracy features are considered as an effort toward the improvement of performance and usability. One can only be improved at the cost of the other, leading to a new trade-off called interpretability-accuracy trade-off. On the other hand, the use of interval type-2 fuzzy sets in the development of fuzzy classifier is another dimension of improving it. In this paper, a fuzzy classifier named Engineering Student-Fuzzy Classification System (ES-FCS) is proposed and implemented using type-1 and type-2 fuzzy logic. The accuracy improvement has been studied by the application of linguistic hedges. The interpretability and accuracy assessment and their trade-off are experimentally studied in evolutionary multi-objective framework with both type- 1 and type- 2 fuzzy sets.
\end{abstract}

Keywords: fuzzy systems; interpretability-accuracy trade-off; genetic fuzzy systems; GFSs; genetic algorithms; GAs; multi-objective evolutionary algorithms; MOEA.

Reference to this paper should be made as follows: Shukla, P.K. and Tripathi, S.P. (2016) 'Interpretability and accuracy issues in evolutionary multi-objective fuzzy classifiers', Int. J. Soft Computing and Networking, Vol. 1, No. 1, pp.55-69.

Biographical notes: Praveen Kumar Shukla is working as the Head of Department at the Department of Information Technology, Babu Banarasi Das Northern India Institute of Technology, Lucknow, India. He holds a Bachelor of Technology in the Information Technology and Master of Technology in Computer Science and Engineering. He is also a PhD student at Department of Computer Science and Engineering, Uttar Pradesh Technical University, Lucknow, India. His research area includes evolutionary multi-objective optimisation and fuzzy systems. 
Surya Prakash Tripathi is working as Professor and Head of Department at Institute of Engineering and Technology, Lucknow, India. He holds a $\mathrm{PhD}$ in Computer Science and Engineering. His research area includes databases, operating systems, fuzzy systems and evolutionary multi-objective optimisation.

\section{Introduction}

Fuzzy systems (Wang, 1999) are highly capable to deal with imprecision and uncertainty existing in the linguistic information during the modelling of real world applications and are applicable in control, classification and modelling areas. The fuzzy systems are capable to simulate the human reasoning and decision making along with their adaptive behaviour. In the fuzzy systems, more precisely fuzzy knowledge base systems (FKBS) have the knowledge about the problem, encoded in the form of fuzzy if-then rules based on either expert knowledge encoding or using some set of data/machine learning approach.

During the development of fuzzy systems, two important aspects are considered,

1 interpretability (Cassilas et al., 2003a; Gacto et al., 2011; Shukla and Tripathi, 2013)

2 accuracy (Cassilas et al., 2003b).

Interpretability is the quality of modelled system which shows if its behaviour is human understandable or not by seeing its functioning. On the other hand, accuracy is the feature in which the closeness between real and modelled system is judged. Accuracy and interpretability features are contradictory with each other; one can be improved at the cost of the other. This is called the interpretability-accuracy trade-off (Shukla and Tripathi, 2011, 2012a).

In the subsequent progress of developing the fuzzy systems, genetic algorithms (GAs) (Holland, 1975) are well utilised and leads to a new scope of research called, genetic fuzzy systems (GFSs) (Cordon et al., 2001; Herrera, 2005; Cordon et al., 2004; Herrera, 2008). Various components of fuzzy systems are optimised, learned and adapted using GAs.

Evolutionary multi-objective optimisation (Deb, 2001) is used to deal with well known interpretability-accuracy trade-off and results in a new area called 'evolutionary multi-objective fuzzy systems (EMOFS)' (Ishibuchi, 2007; Ducange and Marcelloni, 2011; Fazzolari et al., 2012; Shukla and Tripathi, 2012b).

The new dimension to improve the FKBS design is the use of type-2 fuzzy logic (Mizumoto and Tanaka, 1976; Mendel and John, 2002) and interval type-2 fuzzy logic (Wu and Mendel, 2002; Wu, 2012). Use of type-2 fuzzy sets make the system computationally expensive and reduction in interpretability. To overcome with this situation, the authors propose the use of interval type-2 fuzzy sets to develop the evolutionary multi-objective fuzzy classifier engineering student-fuzzy classification system ('ES-FCS'). Interval type-2 fuzzy sets are less computationally expensive and have most of the advantages of the type-2 fuzzy sets.

In this paper the authors have proposed 'engineering student fuzzy classification system (ES-FCS)' on which multi-objective evolutionary algorithms-based (MOEA) 
studies have been carried out dealing with interpretability-accuracy trade-off as well as interpretability assessment at different levels. Also, accuracy improvement has been studied with the integration of linguistic hedges.

This paper continues with Section 2 that introduces and reviews application of MOEA in fuzzy systems. Section 3 is the introduction and review of applying linguistic hedges in simple and multi-objective GFSs. The proposed system EC-FCS is discussed in Section 4. Experimental details and results are carried out in Section 5. Section 6 is the conclusion of the paper.

\section{Type-2 fuzzy theory and systems}

A type-2 fuzzy set has one more dimension to deal with the uncertainty not handled in type-1 simple fuzzy sets. Type-2 fuzzy sets are computationally expensive, hence interval type-2 fuzzy sets are used to design the FKBS. In type-2 fuzzy sets, the membership degree is represented by a fuzzy set whereas the interval type- 2 fuzzy sets have an interval for the membership degree and results into less computational cost.

Type-2 fuzzy set is represented by $\mathrm{A}^{*}$ and defined by membership function $\mu_{A^{*}}(x, u)$ where $x \in X$ and $u \in J_{x} \subseteq[0,1]$

$$
A^{*}=\left\{(x, u), \mu_{A^{*}}(x, u) \mid \forall x \in X, \forall u \in J_{x} \subseteq[0,1]\right\}
$$

Here, $0 \leq \mu_{A^{*}}(x, u) \leq 1$ when all $\mu_{A^{*}}(x, u)=1$ then $A^{*}$ is called interval type-2 fuzzy set. The interval type-2 fuzzy set is represented as follows:

$$
\left.A^{I}=\{(x, u), 1\} \mid x \in X, u \in J_{x}, J_{x} \subseteq[0,1]\right\}
$$

In the above definitions, $X$ is considered as primary domain and $J_{x}$ is the secondary domain. Here, all secondary grades $\mu_{A^{I}}(x, u)$ are equal to 1 . This is fully expressed by the upper and lower bound.

\section{Integration of evolutionary multi-objective optimisation in fuzzy systems to deal with interpretability-accuracy trade-off}

Evolutionary algorithms are powerful and robust search mechanism based on simulation of the concept of natural evolution. These are integrated with multi-objective optimisation leading to a new kind of algorithms, denoted by MOEA. The first generation MOEAs are; non-dominated sorting genetic algorithm (NSGA) (Srinivas and Deb, 1994), niched Pareto genetic algorithms (NPGA) (Horn et al., 1994), multi-objective genetic algorithm (MOGA) (Fonseca and Fleming, 1993) and second generation MOEA are; strength Pareto evolutionary algorithms (SPEA) (Zitzler and Thiele, 1999), strength Pareto evolutionary algorithm 2 (SPEA2) (Zitzler et al., 2001), Pareto archived evolutionary strategies (PAES) (Knowles and Corne, 2000), non-dominated sorting genetic algorithms (NSGA-II) (Deb et al., 2002), niched Pareto genetic algorithm II (NPGA-II) (Erickson et al., 2001) and Pareto envelop-based selection algorithm (PESA) (Corne et al., 2000). 
In EMO development environment, the fuzzy systems are characterised by two conflicting objective functions. These are as follows;

minimise error $\left(f_{S}\right)$ and complexity $\left(f_{S}\right)$

maximise accuracy $\left(f_{s}\right)$ and complexity $\left(f_{s}\right)$

Here, $f s$ is a fuzzy system.

These approaches of integrating GAs with fuzzy system are generating only one fuzzy system on the curve derived in between the accuracy and interpretability. But, further research in this field has proceeded in the new dimension of finding multiple number of fuzzy systems on the curve of accuracy and interpretability. One of these fuzzy systems may be selected as per the preference of user satisfying the problem state. This new dimension leads to a new field, called EMOFS as discussed in Figure 1.

Figure 1 Single and multi-objective GFSs (see online version for colours)

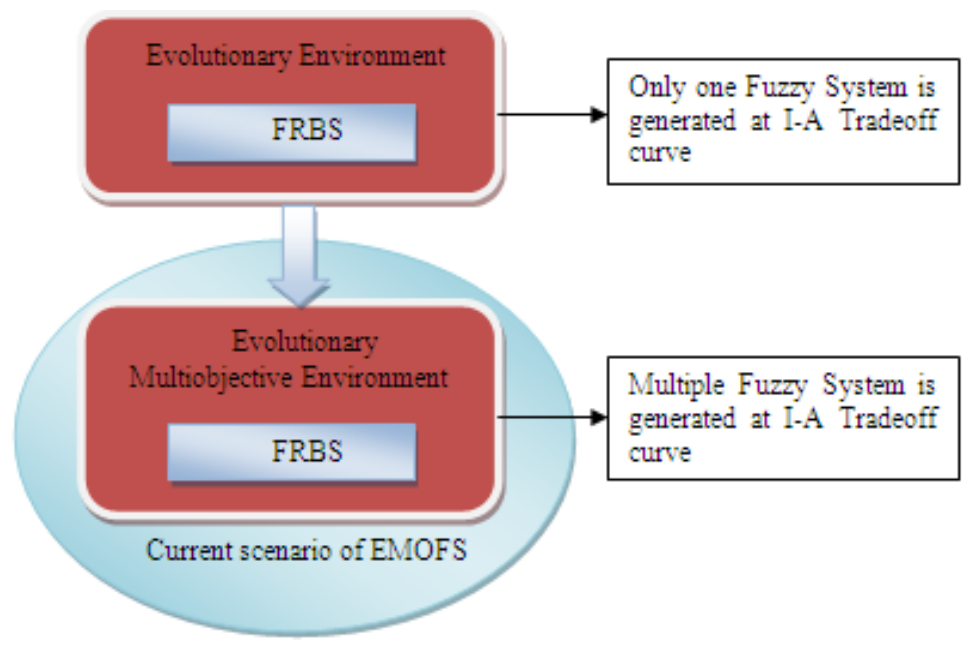

The simple changes in the multi-objective formulation of fuzzy system design are studied in Ishibuchi et al. (2010)

To deal with interpretability and accuracy parameters several multi-objective formulations are developed. In Ishibuchi et al. (1997), the two objectives are; number of correctly classified training patterns (accuracy) and number of selected fuzzy rules (interpretability). In Ishibuchi et al. (2001), the above formulation has been extended with one another objective. The objectives in this formulation are; number of correctly classified training patterns, number of selected fuzzy rules and total number of antecedent conditions [total rule length (TRL)].

Error and complexity are represented by many parameters, like error may be expressed by mean square error (MSE) and root mean square error (RMSE) and interpretability can be expressed by TRL, number of rules (NOR) and average rule length (ARL). 
Nauck's (2003) index (NI) has been proposed for the assessment of the interpretability of fuzzy rule-based classifiers. It can be defined as follows;

$$
I_{\text {Nauck }}=\text { comp } \times \text { part } \times \text { cov }
$$

Here, comp - number of classes/total number of premises (it measures the complexity),

$$
\text { part }=\frac{1}{\text { number of labels }-1}
$$

(it is average normalised partition index),

$c o v$ is the average normalised coverage degree of the fuzzy partition which is equal to 1 for strong fuzzy partition (SFP).

A global fuzzy index has been introduced in Alonso et al. (2006) and its next version has been discussed in Alonso et al. (2008). In this approach the index has been computed as the output of inference of hierarchical fuzzy system.

\section{Linguistic hedges}

The linguistic hedges are used and applied on the tuned MFs as discussed in Venturini (1993). The main linguistic hedges are very, more-or-less, extremely, very-very, positively, negatively. Linguistic hedges are playing the role of adjective and adverbs in the languages, responsible to change the qualitative statements.

Several linguistic hedges are proposed and used in the literature. For the basic knowledge of readers these are summarised in Table 1 based on the Zadeh interpretations (Shi and Ward, 2001) and see Venturini (1993) and Zadeh (1972) for more details.

\begin{tabular}{|c|c|}
\hline Name of linguistic hedges & Mathematical definition \\
\hline Very & $\begin{array}{l}\mu_{M F}^{\text {very }}(x)=\left(\mu_{M F}(x)\right)^{2} \\
\mu_{M F}^{v e r y}(x)=\left(\mu_{M F}(x)\right) \exp ^{\left(\mu_{M F}(x)-1\right)}\end{array}$ \\
\hline Extremely & $\mu_{M F}^{\text {extremely }}(x)=\left(\mu_{M F}(x)\right)^{3}$ \\
\hline Very very & $\mu_{M F}^{\text {very very }}(x)=\left(\mu_{M F}(x)\right)^{4}$ \\
\hline More-or-less & $\begin{array}{l}\mu_{M F}^{\text {more-or-less }}(x)=\left(\mu_{M F}(x)\right)^{0.5} \\
\mu_{M F}^{\text {more-or-less }}(x)=\left(\mu_{M F}(x)\right)^{1 / p} \quad \text { where } p>1\end{array}$ \\
\hline Positively & $\mu_{M F}^{\text {positively }}(x)=\left\{\begin{array}{c}2\left(\mu_{M F}(x)\right)^{2} \text { if } \mu_{M F}(x)<0.5 \\
1-2\left(\mu_{M F}(x)\right)^{2} \text { if } \mu_{M F}(x) \geq 0.5\end{array}\right.$ \\
\hline Negatively & $\mu_{M F}^{\text {negatively }}(x)=\left\{\begin{array}{cl}2\left(\mu_{M F}(x)\right)^{2} & \text { if } \mu_{M F}(x)<0.5 \\
1-(0.5)^{1-1 / n}\left(1-\mu_{M F}(x)\right)^{1 / n} & \text { if } \mu_{M F}(x) \geq 0.5\end{array}\right.$ \\
\hline
\end{tabular}

Table 1 Linguistic hedges 


\section{Proposed system}

\subsection{Engineering student fuzzy classification system}

The authors propose 'ES-FCS'. This is the fuzzy classification system that classifies the students of one class into four groups. These four groups are denoted by 1, 2, 3 and 4 numerical digits with following description given in Table 2.

Table 2 Classification of students

\begin{tabular}{lccc}
\hline 1 & 2 & 3 & 4 \\
Excellent & Good & Average & Poor \\
\hline
\end{tabular}

The classification uses three parameters to take decisions for a student to classify him/her in a particular class. The parameters are;

1 internal assessment (IA)

2 external assessment (EA)

3 attendance (AT)

The descriptions of the above parameters are as follows:

1 Internal assessment: In this assessment, the students are appearing in the internal examination in their department. Three examinations are conducted in the whole semester on the approximate competition of $30 \%, 60 \%$ and $100 \%$ completion of the syllabus. Finally the sessional marks of each subject are awarded on behalf of the marks achieved by the students and the assignments/ tutorial sheets submitted to the subject teacher along with the attendance in that particular subject.

2 External assessment: This assessment is basically the external examination conducted by the affiliating university at the end of the semester. The examinations have subjective paper in each subject with full syllabus. The preparation of the question paper and evaluation of answer sheets are conducted by the affiliating university.

3 Attendance: Attendance is an important criteria to judge the sincerity and hardworking of the student. So attendance $\%$ is calculated at the end of the semester in all theory and lab sessions. The block diagram of the proposed fuzzy classifier is given in Figure 2.

Figure 2 Proposed student classification system

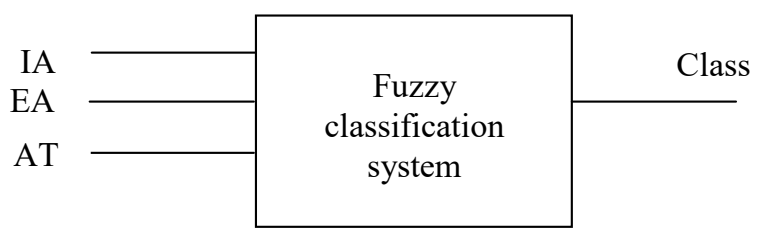




\subsection{Type-1 fuzzy set implementation}

The membership functions of all three assessment parameters are shown in Figures 3 to 5 .

Figure 3 MF of internal assessment (IA) (see online version for colours)
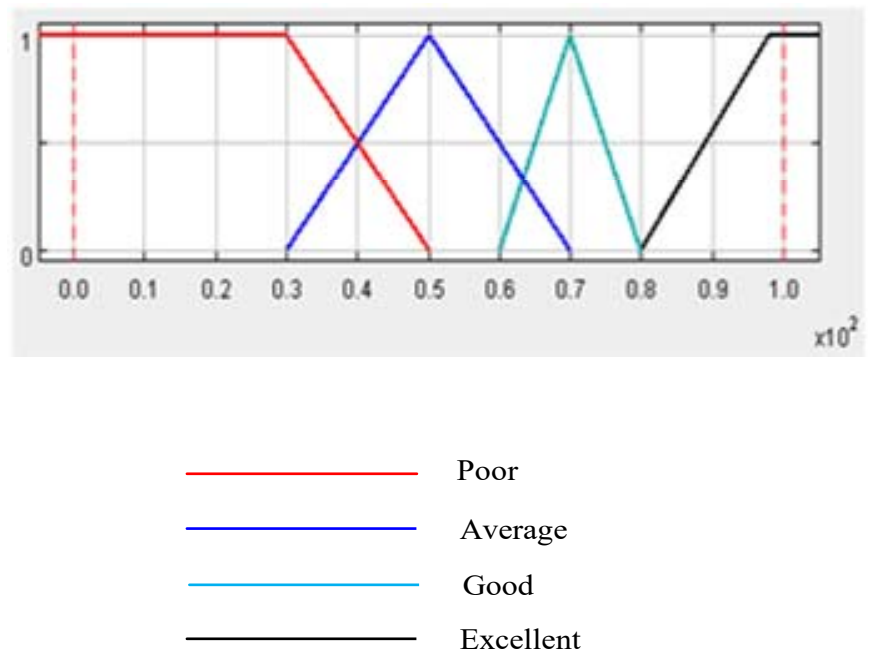

Note: The above linguistic values are applicable on all performance assessment parameters.

Figure 4 MF of external assessment (EA) (see online version for colours)

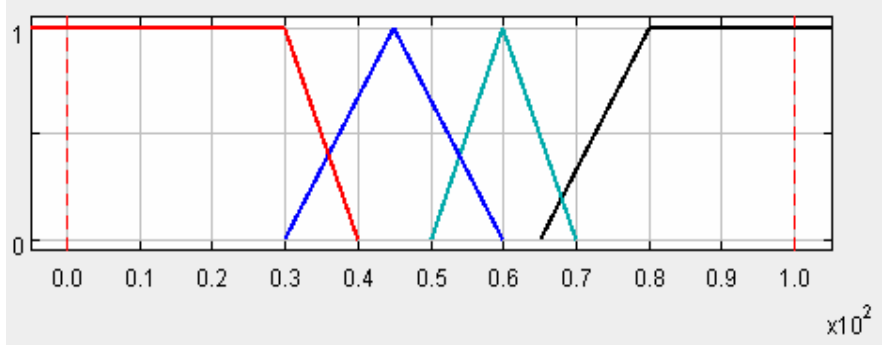

Figure 5 MF of attendance (AT) (see online version for colours)

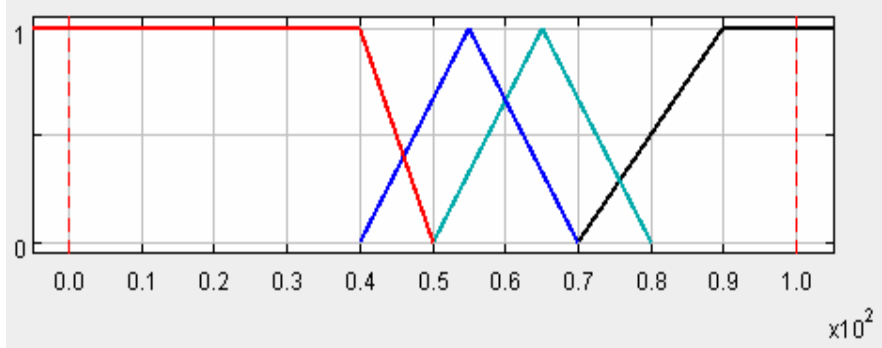




\subsection{Interval type-2 fuzzy set implementation}

The interval type-2 fuzzy sets (Shukla and Tripathi, 2014b) are used to implement the proposed system. The used membership function is given in Figure 6.

Figure 6 Interval type-2 fuzzy set

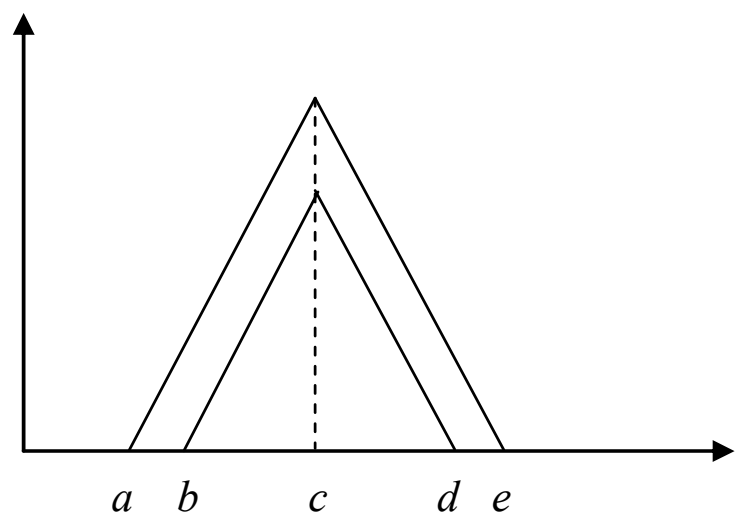

In GAs, the membership function is represented as follows;

Figure 7 Chromosome representing membership function of interval type-2 fuzzy set (see online version for colours)

\begin{tabular}{|l|l|l|l|l|}
$a$ & $b$ & $c$ & $d$ & $e$ \\
\hline
\end{tabular}

If the rule $R 1$ is as follows:

$$
\text { if } x_{1} \text { is } M F_{1}, x_{2} \text { is } M F_{2}, \ldots \ldots . . \text { and } x_{i} \text { is } M F_{i} \text { then } y \text { is } M F_{i+1}
$$

the encoding of the RB is expressed as given in Figure 8.

Figure 8 Chromosome of knowledge base representation (see online version for colours)

\begin{tabular}{|c|c|c|c|c|c|c|c|}
\hline$a_{1}$ & $b_{1}$ & $c_{1}$ & $d_{1}$ & $e_{1}$ & $a_{2}$ & $b_{2}$ & $c_{2}$ \\
\hline$d_{2}$ & $e_{2}$ & \multicolumn{2}{|c}{$\ldots \ldots \ldots \ldots \ldots}$. & $a_{i}$ & $b_{i}$ & $c_{i}$ & $d_{i}$ \\
\hline$e_{i}$ & $a_{i+1}$ & $b_{i+1}$ & $c_{i+1}$ & $d_{i+1}$ & $e_{i+1}$ & \multicolumn{2}{|c}{} \\
\cline { 1 - 5 }
\end{tabular}

The rule base is represented by decision tree using Thrift's approach (Thrift, 1991) as given in Figure 9.

Let $\mathrm{A} 1$ and $\mathrm{A} 2$ are the input variables and the output $\mathrm{Y}$ is as given below.

$$
Y=\{E, G, A\}
$$


Figure 9 Decision table for knowledge base (see online version for colours)

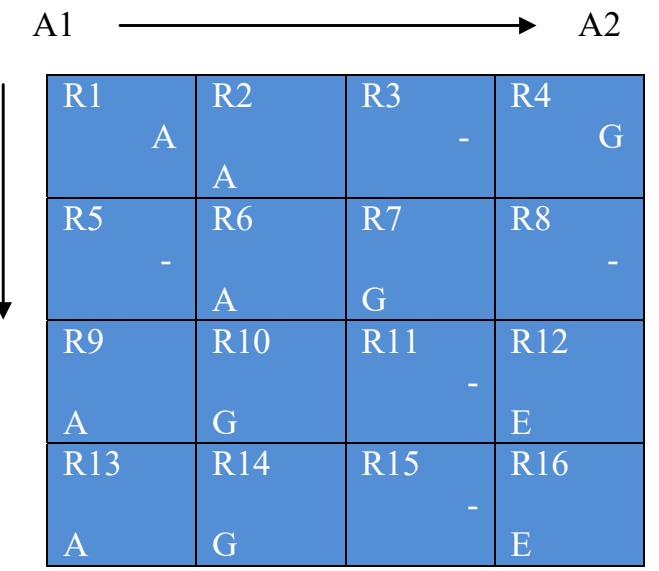

The output is encoded as given in Figure 10.

Figure 10 Output encoding

\begin{tabular}{|c|c|c|}
\hline $\mathrm{E}$ & $\mathrm{G}$ & $\mathrm{A}$ \\
\hline 1 & 2 & 3 \\
\hline
\end{tabular}

The encoded rule base is expressed by Figure 11.

Figure 11 Encoded rule base (see online version for colours)

\begin{tabular}{|l|l|l|l|l|l|l|}
\hline 0 & 3 & 0 & 2 & 0 & 3 & 2 \\
\hline 0 & 3 & 0 & 2 & 1 & 3 & 2 \\
\hline 0 & 1 &
\end{tabular}

The interval type-2 fuzzy membership functions are given in Figures 12 to 14.

Figure 12 MF of internal assessment (see online version for colours)

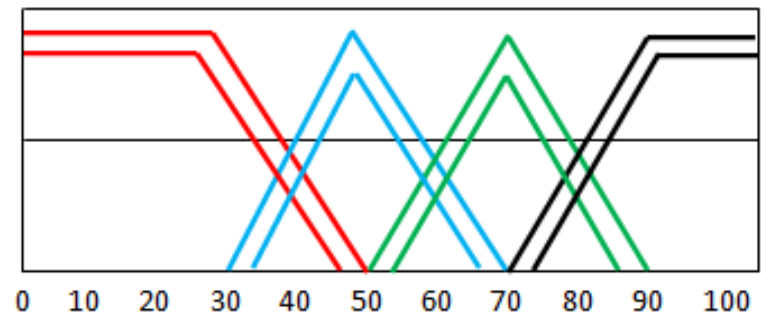


Figure 13 MF of external assessment (see online version for colours)

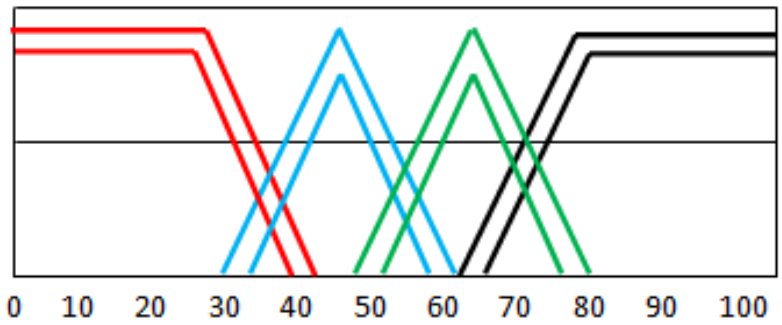

Figure 14 MF of attendance (see online version for colours)

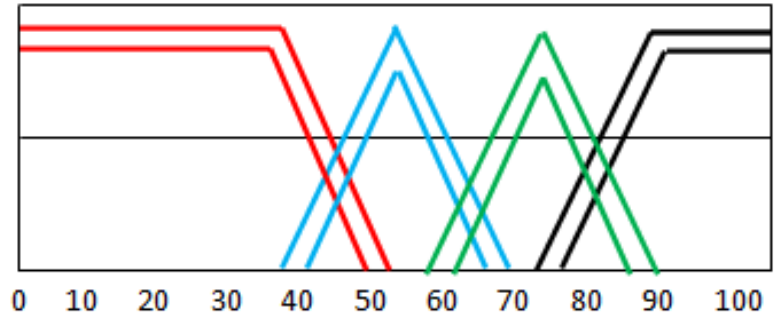

\section{Experiments and result analysis}

The proposed ES-FCS has been implemented using the open access software Guaje (Alonso and Magdalena, 2011a, 2011b) with type-1 fuzzy implementation. The EMO experimentations are carried out with the help of MATLAB. The interpretability of the proposed system is assessed in terms of NOR, TRL, ARL, average fired rules (AFR) and NI. On the other hand, accuracy is measured in terms of percentage of correctly classified students (PCCS).

\subsection{Type-1 implementation}

The results of interpretability and accuracy on different values of NOR are given in Table 3.

Table 3 Interpretability and accuracy parameters

\begin{tabular}{lccccccc}
\hline \multirow{2}{*}{ Experiments } & \multicolumn{5}{c}{ Interpretability } & & Error \\
\cline { 2 - 5 } & Nauck's index & NOR & TRL & ARL & AFR & & PCCS tst $_{n}$ \\
\hline E1 & 0.056 & 8 & 24 & 3 & 3.50 & $71.4 \%$ \\
E2 & 0.037 & 12 & 36 & 3 & 4.67 & $71.4 \%$ \\
E3 & 0.026 & 17 & 51 & 3 & 7.92 & $76.9 \%$ \\
E4 & 0.021 & 21 & 63 & 3 & 7.41 & \\
E5 & 0.018 & 25 & 73 & 2.92 & 9.11 & \\
E6 & 0.016 & 29 & 85 & 2.93 & 9.50 & \\
\hline
\end{tabular}


On the different input MFs, the effect of using linguistic hedges has been studied. The linguistic hedges are; more-or-less and strictly. The linguistic modifiers have improved the accuracy as per the results in Table 4 .

Table 4 Comparative results: linguistic hedges

\begin{tabular}{lcccc}
\hline Condition & Accuracy & Nauck Index & NOR & TRL \\
\hline Without LH & $80.9 \%$ & 0.021 & 21 & 63 \\
With LH & $84.1 \%$ & 0.021 & 21 & 63 \\
\hline
\end{tabular}

Note: $\mathrm{LH}$ - linguistic hedges

As discussed in Alcala et al. (2009) and Shukla and Tripathi (2014a), the multiobjective formulations are as follows:

$g_{1}(x)=$ Percentage error in correctly classified students (PECS)

$g_{2}(x)=$ Number of rules $(N O R)$

$g_{3}(x)=$ Total rule length $(T R L)$

- formulation 1:

minimise $P E C S$ and minimise $N O R$

- formulation 2:

minimise PECS and minimise $T R L$

The single objective maximisation formulations are as follows:

- formulation 1:

$$
\begin{aligned}
& f_{1}(S)=g_{1}(S)-w_{1} g_{2}(S) \\
& f_{2}(S)=g_{2}(S)-w_{1} g_{1}(S)
\end{aligned}
$$

- formulation 2:

$$
\begin{aligned}
& f_{1}(s)=g_{1}(x)-w_{2} g_{3}(x) \\
& f_{2}(s)=g_{3}(x)-w_{2} g_{1}(x)
\end{aligned}
$$

The non-dominated solution as per the formulation 1 and 2 are shown in Figures 15 and 16.

It is to be noted that the formulations are indirectly representing

minimise error and minimise interpretability

The error is measured in terms of PECS and interpretability is measured in terms of NOR and TRL. 
Figure 15 Pareto front with formulation 1 (see online version for colours)

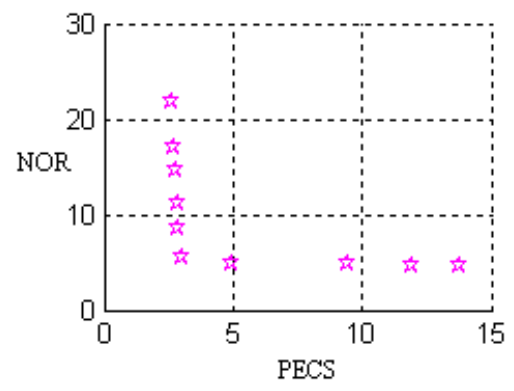

Figure 16 Pareto front with formulation 2 (see online version for colours)

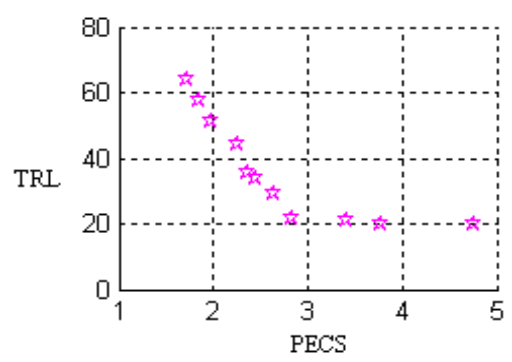

\subsection{Type-2 implementation}

The results of interpretability and accuracy on different values of NOR are given in Table 5.

Table 5 Interpretability and accuracy parameters

\begin{tabular}{|c|c|c|c|c|c|}
\hline \multirow{2}{*}{ Experiments } & \multicolumn{4}{|c|}{ Interpretability } & \multirow{2}{*}{$\frac{\text { Accuracy }}{\text { PCCS }_{\text {tst }}}$} \\
\hline & NOR & $T R L$ & $A R L$ & $A F R$ & \\
\hline E1 & 8 & 24 & 3 & 3.69 & $70.3 \%$ \\
\hline E2 & 12 & 36 & 3 & 4.89 & $72.3 \%$ \\
\hline E3 & 17 & 51 & 3 & 8.15 & $77.8 \%$ \\
\hline E4 & 21 & 63 & 3 & 7.98 & $82.7 \%$ \\
\hline E5 & 25 & 73 & 2.92 & 10.15 & $91.6 \%$ \\
\hline E6 & 29 & 85 & 2.93 & 10.97 & $94.5 \%$ \\
\hline
\end{tabular}

\section{Conclusions}

Interpretability and accuracy are the important features to be considered during the development of fuzzy systems. The trade-off between these two features is handled by evolutionary multi-objective optimisation of fuzzy systems. In this paper the authors have developed a new 'engineering student-fuzzy classification system (ES-FCS)' in evolutionary multi-objective optimisation framework. The proposed system is 
implemented using type-1 and interval type-2 fuzzy sets. The interpretability and accuracy measures are evaluated and compared in both implementation of type- 1 and interval type 2 fuzzy set. The required Pareto fronts are also generated.

\section{References}

Alcala, R., Ducange, P., Herrera, F., Lazzerini, B. and Marcelloni, F. (2009) 'A multiobjective evolutionary approach to concurrently learn rule and data bases of linguistic fuzzy rule-based systems', IEEE Transactions on Fuzzy Systems, Vol. 17, No. 5, pp.1106-1122.

Alonso, J.M. and Magdalena, L. (2011a) Generating understandable and accurate fuzzy rule based systems in a java environment', Lecture Notes in Artificial Intelligence-9th International Workshop on Fuzzy Logic and Applications, LNAI, Vol. 6857, pp.212-219.

Alonso, J.M. and Magdalena, L. (2011b) 'HILK++: an interpretability guided fuzzy modeling methodology for learning readable and comprehensible fuzzy rule based classifiers', Soft Computing, Vol. 15, No. 10, pp.1959-1980.

Alonso, J.M., Guillaume, S. and Magdalena, L. (2006) 'A hierarchical fuzzy system for assessing interpretability of linguistic knowledge bases in classification problems', in Proceedings of IPMU, Information Processing and Management of Uncertainty in Knowledge Based Systems, July $2-7$, pp.348-355.

Alonso, J.M., Guillaume, S. and Magdalena, L. (2008) 'HILK: a new methodology for designing highly interpretable linguistic knowledge bases using the fuzzy logic formalism', International Journal of Intelligent Systems, Vol. 23, No. 7, pp.761-794.

Cassilas, J., Cordon, O. and Herrera, F. (2003a) Interpretability Improvements in Linguistic Fuzzy Modelling, Springer, Heidelberg, Germany.

Cassilas, J., Cordon, O., Herrera, F. and Magdalena, L. (2003b) Accuracy Improvements in Linguistic Fuzzy Modelling, Springer, New York, USA.

Cordon, O., Gomide, F., Herrera, F., Hoffmann, F. and Magdalena, L. (2004) 'Ten years of genetic fuzzy systems: current framework and new trends', Fuzzy Sets and Systems, Vol. 141, No. 1, pp.5-31.

Cordon, O., Herrera, F., Hoffmann, F. and Magdalena, L. (2001) Genetic Fuzzy Systems: Evolutionary Tuning and Learning of Fuzzy Knowledge Bases, World Scientific, Singapore.

Corne, D.W., Knowles, J.D. and Oates, M.J. (2000) 'The Pareto envelop based selection algorithm for multi-objective optimization', 6th Conference of Parallel Problem Solving from Nature, Paris, France, Springer LNCS 1917, pp.839-848.

Deb, K. (2001) Multiobjective Optimization using Evolutionary Algorithms, John Wiley \& Sons, Chichester, UK.

Deb, K., Pratap, A., Agarwal, S. and Meyarivan, T. (2002) 'A fast and elitist multi-objective genetic algorithm: NSGA II’, IEEE Transactions on Evolutionary Computation, Vol. 6, No. 2, pp.182-197.

Ducange, P. and Marcelloni, F. (2011) Multiobjective Evolutionary Fuzzy Systems, pp.83-90, Springer-Verlag, Berlin/Heidelberg, Germany.

Erickson, M., Mayer, A. and Horn, J. (2001) 'The niched Pareto genetic algorithms applied to the design of ground water remediation system', First International Conference on Evolutionary Multi Criteria Optimization, Springer-Verlag, LNCS, No. 1993, pp.681-695.

Fazzolari, F., Alcala, R., Nojima, Y., Ishibuchi, H. and Herrera, F. (2012) 'A review of the application of multiobjective evolutionary fuzzy systems: current state and further directions', IEEE Transactions on Fuzzy Systems, doi: 10/1109/TFUZZ.2012.2201338.

Fonseca, C.M. and Fleming, P.J. (1993) 'Genetic algorithms for multiobjective optimization: formulation, discussion and generalization', 5th International Conference on Genetic Algorithms, pp.416-423. 
Gacto, M.J., Alcala, R. and Herrera, F. (2011) 'Interpretability of linguistic fuzzy rule based systems: an overview of interpretability measures', Information Sciences, Vol. 181, No. 20, pp.4340-4360.

Herrera, F. (2005) 'Genetic fuzzy systems: status, critical considerations and future directions', International Journal of Computational Intelligence Research, Vol. 1, No. 1, pp.59-67.

Herrera, F. (2008) 'Genetic fuzzy systems: taxonomy, current research trends and prospects', Evolutionary Intelligence, Vol. 1, pp.27-46.

Holland, J.H. (1975) Adaptation in Natural and Artificial Systems, University of Michigan Press, Ann Arbor.

Horn, J., Nafpliotis, N. and Goldberg, D.E. (1994) 'A niched Pareto genetic algorithm for multiobjective optimization', in Proc.: 1st IEEE Conference on Evolutionary Computation. IEEE World Congress on Computational Intelligence, Vol. 1, pp.82-87.

Ishibuchi, H. (1997) 'Single objective and two objective genetic algorithms for selecting linguistic rules for pattern classification problems', Fuzzy Sets and Systems, Vol. 89, No. 2, pp.135-150.

Ishibuchi, H. (2007) 'Multiobjective genetic fuzzy systems: review and future research directions', FUZZ-IEEE, London, UK, 23-26 July, pp.913-918.

Ishibuchi, H., Nakashima, T. and Murata, T. (2001) 'Three-objective genetics-based machine learning for linguistic rule extraction', Information Sciences, Vol. 136, Nos. 1-4, pp.109-133.

Ishibuchi, H., Nakashima, Y. and Nojima, Y. (2010) 'Simple changes in problem formulations make a difference in multiobjective genetic fuzzy systems', 4th International Workshop on Genetic and Evolutionary Fuzzy Systems, Mieres, Spain, March, pp.3-8.

Knowles, J.D. and Corne, D.W. (2000) 'Approximating the non-dominated front using the Pareto achieved evolution strategy', Evolutionary Computation, Vol. 8, No. 2, pp.149-172.

Mendel, J.M. and John, R.I.B. (2002) 'Type-2 fuzzy sets made simple', IEEE Transactions on Fuzzy Systems, Vol. 10, No. 2, pp.117-127.

Mizumoto, K. and Tanaka, K. (1976) 'Some properties of fuzzy sets of type-2', Information Control, Vol. 31, No. 4, pp.312-340.

Nauck, D.D. (2003) 'Measuring interpretability in rule based classification systems', FUZZ-IEEE, May 25-28, pp.196-201.

Shi, H. and Ward, R. (2001) 'Expanding the definitions of linguistic hedges', in Proceedings of Joint 9th IFSA World Congress \& 20th NAFIPS, pp.2591-2595.

Shukla, P.K. and Tripathi, S.P. (2011) 'A survey on interpretability-accuracy trade-off in evolutionary fuzzy systems', 5th International Conference on Genetic and Evolutionary Computing, Taiwan, Xiamen, pp.97-101.

Shukla, P.K. and Tripathi, S.P. (2012a) 'On the design of interpretable evolutionary fuzzy systems (I-EFS) with improved accuracy', International Conference on Computing Sciences, pp.11-14.

Shukla, P.K. and Tripathi, S.P. (2012b) 'A review on the interpretability-accuracy trade-off in evolutionary multiobjective fuzzy systems (EMOFS)', Information, Vol. 3, No. 3, pp.256-277.

Shukla, P.K. and Tripathi, S.P. (2013) 'Interpretability issues in evolutionary multi-objective fuzzy knowledge base systems', J.C. Bansal (Eds.): Proceedings of 7th International Conference on Bio-Inspired Computing: Theories and Applications (BICTA-2012), Advances in Intelligent Systems and Computing, Vol. 201, pp.473-484.

Shukla, P.K. and Tripathi, S.P. (2014a) 'Handling high dimensionality and interpretability-accuracy trade-off issues in evolutionary multi-objective fuzzy classifiers', International Journal of Scientific and Engineering Research, Vol. 5, No. 6, pp.665-670.

Shukla, P.K. and Tripathi, S.P. (2014b) 'A new approach for tuning interval type-2 fuzzy knowledge bases using genetic algorithms', Journal of Uncertainty Analysis and Applications, Vol. 2, No. 1, pp.1-15. 
Srinivas, N. and Deb, K. (1994) 'Multiobjective optimization using non-dominated sorting in genetic algorithms', Evolutionary Computation, Vol. 2, No. 3, pp.221-248.

Thrift, P. (1991) 'Fuzzy logic synthesis with genetic algorithms', Proc. of 4th International Conference on Genetic Algorithms (ICGA '91), San Diago, USA, pp.509-513.

Venturini, G. (1993) 'SIA: a supervised inductive algorithm with genetic search for learning attribute based concepts', in Proceedings of European Conference on Machine Learning, Viena, pp.280-296.

Wang, L-X. (1999) A Course in Fuzzy Systems and Control, Prentice Hall International, USA.

$\mathrm{Wu}, \mathrm{D}$. (2012) 'On the fundamental differences between interval type-2 and type-1 fuzzy logic controller', IEEE Transactions on Fuzzy Systems, Vol. 20, No. 5, pp.832-848.

Wu, H. and Mendel, J.M. (2002) 'Uncertainty bounds and their use in the design of interval type-2 fuzzy logic systems', IEEE Transactions on Fuzzy Systems, Vol. 10, No. 5, pp.622-639.

Zadeh, L.A. (1972) 'A fuzzy set theoretic interpretation of linguistic hedges', Journal of Cybernetics, Vol. 2, No. 3, pp.4-34.

Zitzler, E. and Thiele, L. (1999) 'Multiobjective evolutionary algorithms: a comparative case study and the strength Pareto approach', IEEE Transactions on Evolutionary Computation, Vol. 3, No. 4, pp.257-271.

Zitzler, E., Laumanns, M. and Thiele, L. (2001) SPEA2: Improving the Strength Pareto Evolutionary Algorithms, Technical Report 103, Computer Engineering \& Networks Laboratory (TIK), Swiss Federal Institute of Technology (ETH), Zurich, Switzerland. 